\title{
Discriminating between Mozart's Symphonies and String Quartets Based on the Degree of Independency between the String Parts
}

\author{
Michiru Hirano* and Hilofumi Yamamoto*
}

\begin{abstract}
This paper aims to demonstrate that variables relating to the independency of the string parts are useful for discriminating between symphonies and string quartets in terms of their orchestrations. More specifically, this paper targets the fast initial movements of thirty-nine symphonies and twenty string quartets by Mozart. Conducted analysis extracted three factors within the twelve observed variables and suggests that one of those factors, namely, the Lower part Pitch-Name and Duration factor (LPND), can account for the differences between Mozart's symphonies and string quartets composed after 1782.
\end{abstract}

\section{Introduction}

It is often difficult to discern differences between musical features within the scores for symphonies and string quartets. The present paper seeks to demonstrate that the variables relating to the independency of the string parts are useful in discriminating between the two genres in terms of their orchestration.

There are some common features to be seen between symphonies and string quartets. Both genres were established during the eighteenth century (Hickman 1981, Hodeir 1973). They usually consist of three or four movements. They have common instrumentation in terms of the string section, which is normally divided into four parts: first violin, second violin, viola, and bass. On the other hand, the two genres differ superficially on the following points. First, the instrumentation of a symphony usually includes wind and percussion sections as well as the string section, while string quartets consist only of strings. Second, in terms of the bass part, while contrabasses, a bassoon, and even a harpsichord are sometimes added to the cellos for symphonies (Zaslaw 2003), the majority of string quartets employ only the cello. Third, the number of string players

\footnotetext{
${ }^{*}$ Department of Value and Decision Science, Tokyo Institute of Technology
} 
Discriminating between Mozart's Symphonies and String Quartets Based on the Degree of Independency between the String Parts

per part differs; each string part is performed by more than one player in the case of symphonies, but it is performed by a single player in the case of string quartets. These different styles of performance are known as orchestral performance and soloistic performance, respectively (Hickman 1981).

In addition to these apparent differences, it has been proposed that there are also distinctions relating to the internal structures of the musical scores, such as texture, dynamics, melody, rhythm, harmony, range of instruments, and idiomatic features (Hickman 1981, table 1). These musical features of scores are regarded as being partially reflected in the performance styles. Soloistic scoring, found in string quartets, enables players to perform with more freedom and flexible expression, while orchestral scoring, found in symphonies, imposes expressive restrictions on the players through the rigors of playing the same part with other players (Hickman 1981). Thus, composers usually apply suitable ways of composing their works for each style (Becker 2013, 45-46). Accordingly, one might expect that it should be possible to objectively discern differences in the orchestrations of the string sections within symphonies and string quartets that reflect their respective performance styles, regardless of similarities in terms of the instrumentations.

We focus on the variables relating to the independency of the string parts, which are indexes of the divergence from the other parts. The independency of a part refers to its prominence within a composition. If a part has a high degree of independency, the part plays an essential role within the composition, because it is frequently different from the other parts; a part with a lower degree of independency is less influential within a composition because it mainly overlaps with other parts. We assume that composers vary the independencies within compositions to match the performance style, because independency relates to how musical materials, such as motifs, are assigned to the parts.

In order to gain objective evidence based on scores that would be applicable for all cases, we introduce a method of analyzing musical scores quantitatively. Although computational methods for extracting the musical features of scores have been proposed (by, e.g., Chai and Vercoe (2001), Cambouropoulos et al. (2005), and Conklin and Bergeron (2007)), to date, no approaches have been developed for comparing symphonies and string quartets based on the independencies of the parts.

This paper focuses on symphonies and string quartets by Wolfgang Amadeus Mozart (1756-1791) to investigate distinctions between the ways Mozart composed in the two different genres. 
Discriminating between Mozart's Symphonies and String Quartets Based on the Degree of Independency between the String Parts

\section{Methods}

\subsection{Materials}

Mozart is one of the most important composers of the classical period, when many symphonies and string quartets were written, and his compositions are often said to reflect the trend of the period (Osaki 2005, 152). Mozart is, therefore, a suitable composer to examine in this context.

It is believed that Mozart composed nearly fifty symphonies, of which thirtynine authentic works exist today (Zaslaw 2003). There are also twenty-three string quartets by Mozart. All of Mozart's symphonies and string quartets consist of three or four movements, like those by other classical composers. The tempo of each movement is written on the score. In most cases, the tempo of the initial movement is "allegro" or "presto" (both meaning "fast"). This type of movement is often regarded as being the representative element of the whole work (Hodeir 1973). Since restricting the type of movements analyzed can contribute to making the comparison results clearer, only the fast, initial movements are analyzed within this study. While all the initial movements of Mozart's symphonies satisfy this condition, three of Mozart's twenty-three string quartets (K. 73f, K. 159, and K. 170) are excluded from the present analyses because the tempos of their initial movements are either "adagio" or "andante" (both meaning "slow"). Thus, we used the materials shown in table 1 . All the scores were obtained from Bärenreiter Verlag's Neue Mozart-Ausgabe (NMA) (New Mozart Edition), which are available online. $^{1}$

1 NMA Online: Neue Mozart-Ausgabe, digitized version. Salzburg, Austria: Internationale Stiftung Mozart; Los Altos, CA: The Packard Humanities Institute, 2006- . Accessed May 17, 2017. http://dme.mozarteum.at/DME/nma/start.php?l=2. 
Discriminating between Mozart's Symphonies and String Quartets Based on the Degree of Independency between the String Parts

Table 1. List of the experimental materials: the initial movements of thirty-nine symphonies and twenty string quartets were examined. The number assigned to each work is from the sixth edition of the Köchel catalogue, which is a chronological catalogue of Mozart's compositions. The composition year for each work is also shown in the table. Three of the string quartets-K. 73f, K. 159, and K. 170-were removed from the materials because the tempos of their initial movements are not fast.

\begin{tabular}{|c|c|c|c|c|c|}
\hline \multicolumn{4}{|c|}{ Symphonies } & \multicolumn{2}{|c|}{ String quartets } \\
\hline Number & Year & Number & Year & Number & Year \\
\hline K. 16 & 1764 & K. $161 \mathrm{a}$ & 1773 & K. $134 \mathrm{a}$ & 1772 \\
\hline K. 19 & 1765 & K. $161 b$ & 1773 & K. $134 b$ & 1772 \\
\hline K. $19 a$ & 1765 & K. 162 & 1773 & K. 157 & 1773 \\
\hline K. 22 & 1765 & K. $162 b$ & 1773 & K. 158 & 1773 \\
\hline K. $45 \mathrm{a}$ & 1766 & K. $173 \mathrm{dA}$ & 1773 & K. $159 a$ & 1773 \\
\hline K. 43 & 1767 & K. $173 \mathrm{~dB}$ & 1773 & K. 168 & 1773 \\
\hline K. 45 & 1768 & K. $186 \mathrm{a}$ & 1774 & K. 169 & 1773 \\
\hline K. 48 & 1768 & K. $186 b$ & 1774 & K. 171 & 1773 \\
\hline K. 73 & 1771 & K. $189 \mathrm{k}$ & 1774 & K. 172 & 1773 \\
\hline K. 74 & 1771 & K. $300 \mathrm{a}$ & 1778 & K. 173 & 1773 \\
\hline K. $75 b$ & 1771 & K. 318 & 1779 & K. 387 & 1782 \\
\hline K. 112 & 1771 & K. 319 & 1779 & K. $417 b$ & 1783 \\
\hline K. 114 & 1771 & K. 338 & 1780 & K. $421 \mathrm{~b}$ & 1783 \\
\hline K. 124 & 1772 & K. 385 & 1782 & K. 458 & 1784 \\
\hline K. 128 & 1772 & K. 425 & 1783 & K. 464 & 1785 \\
\hline K. 129 & 1772 & K. 504 & 1786 & K. 465 & 1785 \\
\hline K. 130 & 1772 & K. 543 & 1788 & K. 499 & 1786 \\
\hline K. 132 & 1772 & K. 550 & 1788 & K. 575 & 1789 \\
\hline K. 133 & 1772 & K. 551 & 1788 & K. 589 & 1790 \\
\hline K. 134 & 1772 & & & K. 590 & 1790 \\
\hline
\end{tabular}


Discriminating between Mozart's Symphonies and String Quartets Based on the Degree of Independency between the String Parts

\subsection{Procedures}

The study procedure is as follows. First, the scores were transcribed into MusicXML, which is a textual representation of the musical notation for digitization and makes it possible to read the musical scores with computer programs. Next, twelve variables, which are defined below, were measured for each work. Parallel analysis was conducted in order to determine the number of factors prior to factor analysis. Factor analysis was then carried out, adopting maximum likelihood estimation and promax rotation, in order to extract the smallest number of potential factors from the twelve observed variables. Factor scores for each work were also obtained. Finally, we examined the relationship between these factor scores and the composition year for each work.

\subsection{Variables}

Although the wind and percussion sections of symphonies are assumed to influence the string section, this study examines only the string sections in comparing the two genres. This enables us to investigate the features within the scope of the string section. The study assumes that the bass part for symphonies is performed on the cello. The Italian names of the four string parts, as Mozart indicated them, are used in the remainder of this paper: violino I (first violin), violino II (second violin), viola (viola), and violoncello (cello).

We introduce the concept of independency and examine whether its related variables can discriminate between symphonies and string quartets. Independency is an index of how frequently one part is independent of the other parts. Thus, the term independent refers to a state where a feature of one part is different from the same feature in all of the other parts at a given point in time. Within this study, three features are taken into consideration: (1) durations of notes, (2) pitch-names, and (3) pitch-changes. These three features correspond to the three major elements of music: rhythm, harmony, and melody. The independencies for all four string parts for all three features - making twelve variables in total-were measured for each work. More detailed definitions of these independencies are provided next.

All the kinds of independency extracted within the current study were calculated according to the following formula:

$$
I_{f}(p)=\frac{1}{L} \sum_{t=0}^{L-1} \chi_{f}(p, t),
$$

where $I_{f}(p)$ represents the independency of feature $f$ for part $p$, and $\chi_{f}(p, t)$ is a characteristic function of the feature $f$ for part $p$ at time $t$. A feature $f$ is an element 
Discriminating between Mozart's Symphonies and String Quartets Based on the Degree of Independency between the String Parts

of the set $F=\{\mathrm{d}, \mathrm{pn}, \mathrm{pc}\}$, which represent duration, pitch-name, and pitch-change, respectively. Part $p$ is an element of the set $P=\{$ violino I, violino II, viola, violoncello\}. Time $t$ is a discrete value, considering the shortest duration of notes or rests appearing within a work as its scale. The length of a work, $L$, is represented in terms of time $t$; a work starts when $t=0$ and ends when $t=L$. We check whether the note of part $p$ at time $t$ is independent based on $\chi_{f}(p, t)$. We do not check when $t=L$ because there are no more notes at that point. Independency, $I_{f}(p)$, is calculated as the arithmetic mean of $\chi_{f}(p, t)$.

The characteristic functions of each feature are defined as follows: First, the characteristic function of duration is defined as

$$
\chi_{d}(p, t)= \begin{cases}1 & (\text { if } \boldsymbol{d}(p, t) \neq O \text { and } \forall q \neq p, \boldsymbol{d}(p, t) \neq \boldsymbol{d}(q, t)), \\ 0 & \text { (otherwise), }\end{cases}
$$

where $q$ is an element of set $P$ as well as $p$. The function $\boldsymbol{d}(p, t)$ is a vector defined as

$$
\boldsymbol{d}(p, t)= \begin{cases}\left(\alpha, t_{c}\right) & \text { (if note at time } t), \\ 0 & \text { (if rest at time } t)\end{cases}
$$

where $\alpha$ represents the duration of the note that starts just at time $t$ or the note that starts last prior to time $t$ if the note continues at the time, and $t_{c}$ represents the passage of time since the note started.

Second, the characteristic function of pitch-name is defined as

$$
\chi_{p n}(p, t)= \begin{cases}1 & \text { (if } \exists k,(n(p, t, k) \neq 0 \text { and } \forall q \neq p, \forall l, n(p, t, k) \neq n(q, t, l)), \\ 0 & \text { (otherwise), }\end{cases}
$$

where $n(p, t, k)$ represents the pitch-name of the $k$ th lowest note of part $p$ at time $t$ and $k, l \in \mathbb{N}$. The pitch-name, $n(p, t, k)$, is an element of the set including pitchnames with distinctions between enharmonic notes (such as $\mathrm{F} \#$ and $\mathrm{Gb}$ ) and 0 which stands for a rest, that is,

$$
n(p, t, k) \in\{C, D, E, F, G, A, B, C \#, D \#, \ldots, C b, D b, \ldots, 0\}
$$

Third, the characteristic function of pitch-change is defined as

$$
\chi_{p c}(p, t)= \begin{cases}1 & (\text { if } c(p, t) \neq 0 \text { and } \forall q \neq p, c(p, t) \neq c(q, t)), \\ 0 & \text { (otherwise), }\end{cases}
$$

where $c(p, t)$ represents the pitch-change of part $p$ at time $t$ defined as

$$
c(p, t)= \begin{cases}\gamma & \text { (if note at time } t \text { and } t \geq 1), \\ 0 \quad \text { (if rest at time } t \text { and } t=0),\end{cases}
$$

where $\gamma \in\{1,0,-1\}$. If the pitch of the highest note that sounds at time $t$ is 
Discriminating between Mozart's Symphonies and String Quartets Based on the Degree of Independency between the String Parts

higher than that of the highest note which sounds last prior to time $t, \gamma=1$. If lower, $\gamma$ $=-1$ and if the same, $\gamma=0$.

\section{Results}

The results of the parallel analysis are presented in figure 1 . The figure indicates the suitable number of factors and components, based on both principal component analysis and factor analysis. We adopt three factors according to the number of triangles above the lower dotted line. The results of factor analysis are presented in table 2, which indicates that the factor loading of the first factor was larger for four variables$I_{p n}$ (viola), $I_{d}$ (viola), $I_{p n}$ (violoncello), and $I_{d}$ (violoncello) - than for the others. Similarly, table 2 indicates that the factor loading of the second factor was larger for four variables- $I_{p c}$ (violino $\left.I\right), I_{p c}$ (violoncello), $I_{p c}\left(\right.$ viola), and $I_{p c}($ violino $I I)$ - and the factor loading of the third factor was larger for the remaining four variables, $I_{p n}($ violino $I), I_{p n}($ violino $I I), I_{d}($ violino $I)$, and $I_{d}($ violino $I I)$.

\section{Parallel Analysis Scree Plots}

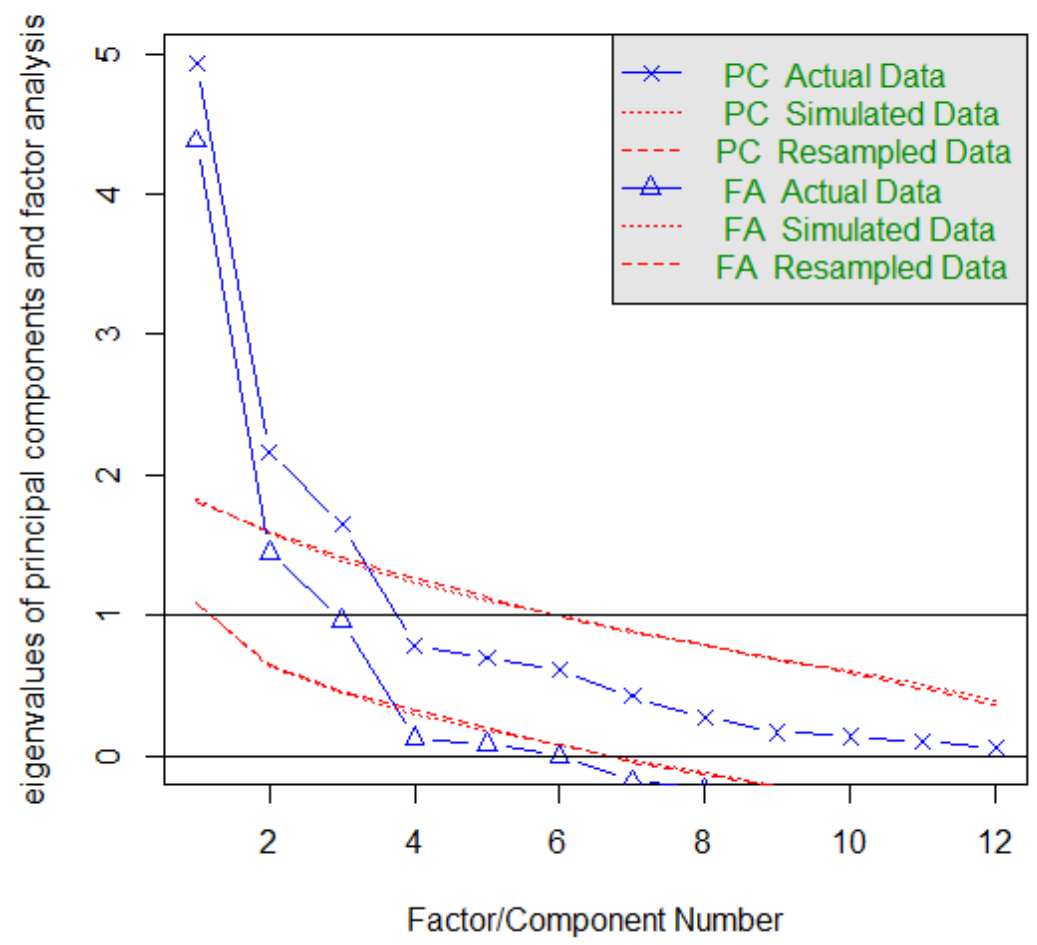

Figure 1. The results of parallel analysis. The number of triangles above the lower dotted line is the number of suitable factors. This figure indicates that the number of suitable factors is three. 
Discriminating between Mozart's Symphonies and String Quartets Based on the Degree of Independency between the String Parts

Table 2. The results of factor analysis. The factor loadings of each factor were larger for four specific variables compared to the others.

\begin{tabular}{|c|c|c|c|c|}
\hline & 1 st factor & 2nd factor & 3rd factor & Commonness \\
\hline$I_{p n}($ viola $)$ & 0.955 & 0.092 & 0.022 & 0.992 \\
\hline$I_{d}($ viola $)$ & 0.809 & -0.111 & -0.133 & 0.547 \\
\hline$I_{p n}($ violoncello $)$ & 0.762 & 0.095 & 0.069 & 0.687 \\
\hline$I_{d}($ violoncello $)$ & 0.652 & -0.008 & 0.053 & 0.456 \\
\hline$I_{p c}($ violino $I)$ & -0.020 & 0.863 & 0.174 & 0.865 \\
\hline$I_{p c}($ violoncello $)$ & 0.080 & 0.829 & -0.175 & 0.650 \\
\hline$I_{p c}($ viola $)$ & 0.297 & 0.781 & -0.149 & 0.734 \\
\hline$I_{p c}($ violino $I I)$ & -0.274 & 0.636 & 0.250 & 0.490 \\
\hline$I_{p n}($ violino $I)$ & -0.106 & 0.011 & 0.969 & 0.864 \\
\hline$I_{p n}($ violino $I I)$ & 0.143 & 0.040 & 0.721 & 0.659 \\
\hline$I_{d}($ violino $I)$ & 0.218 & 0.040 & 0.556 & 0.487 \\
\hline$I_{d}($ violino $I I)$ & 0.084 & -0.158 & 0.468 & 0.228 \\
\hline \multicolumn{5}{|l|}{ Correlations } \\
\hline \multicolumn{5}{|l|}{ between factors } \\
\hline 1 st factor & 1.000 & & & \\
\hline 2nd factor & -0.287 & 1.000 & & \\
\hline 3rd factor & -0.451 & 0.344 & 1.000 & \\
\hline
\end{tabular}

Figures 2 through 4 present the relationships between composition year and the factor scores of each factor. The black circles and gray squares plotted on the graphs indicate symphonies and string quartet compositions, respectively. Figure 2 indicates that the factor scores for symphonies after the year 1773 are negative with the exception of two symphonies in 1788, while the scores for string quartets composed after 1782 are positive. 
Discriminating between Mozart's Symphonies and String Quartets Based on the Degree of Independency between the String Parts

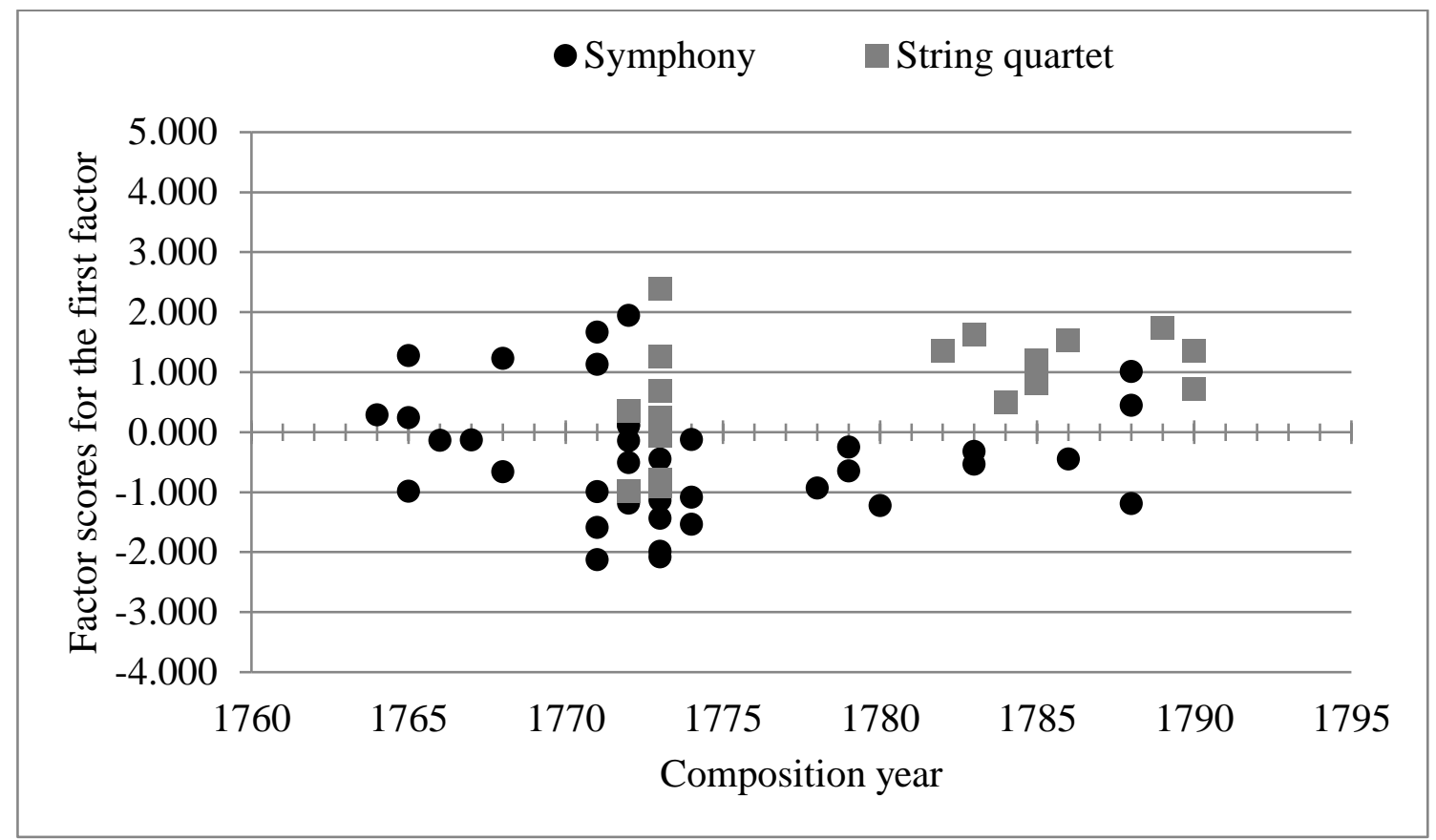

Figure 2. The relationship between composition year (horizontal axis) and factor scores for the first factor (vertical axis). The black circles and gray squares plotted on the graphs indicate symphonies and string quartet compositions, respectively. There is a borderline between the two genres after the year 1782.

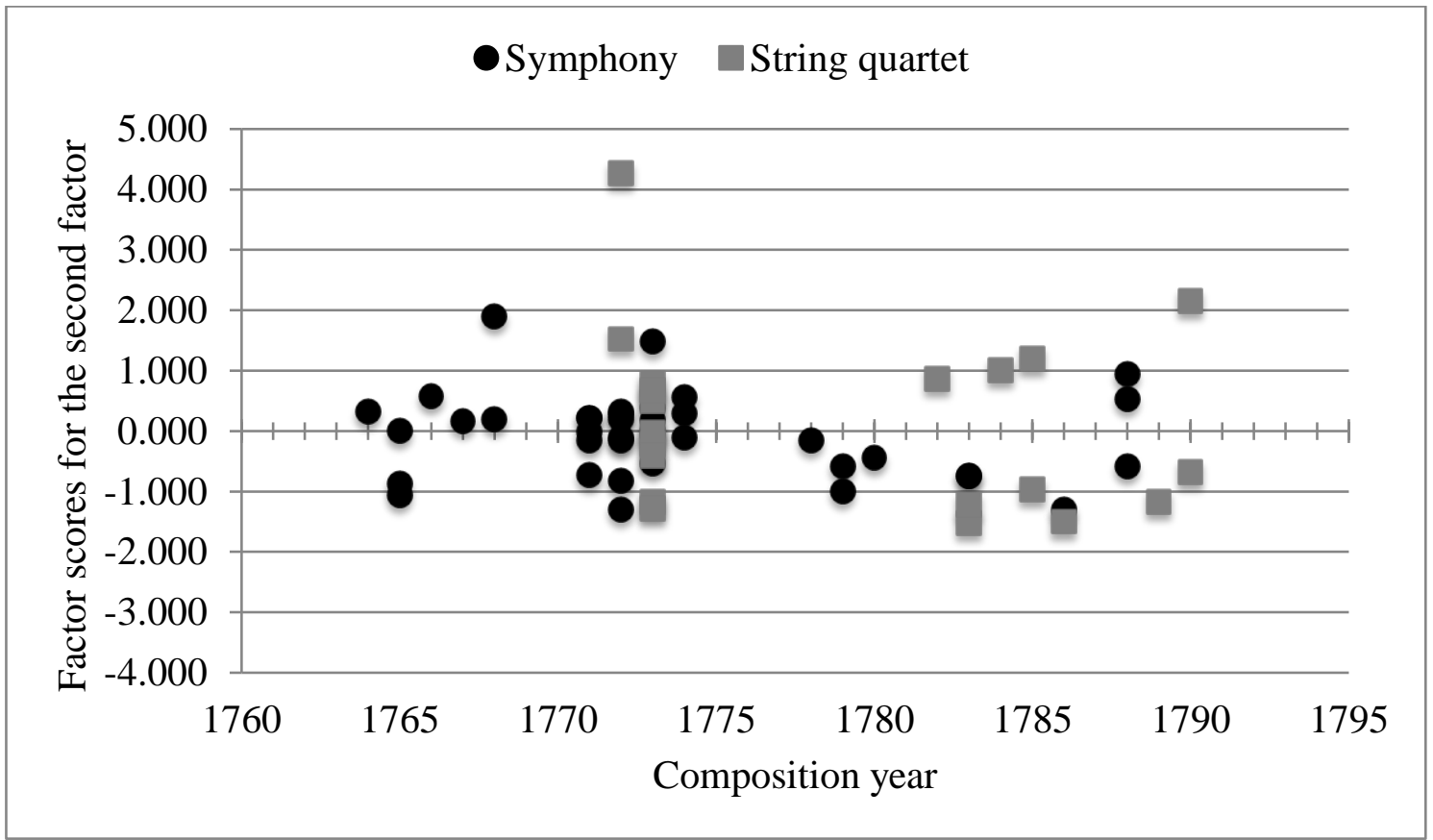

Figure 3. The relationship between composition year (horizontal axis) and the factor scores for the second factor (vertical axis). The black circles and gray squares plotted on the graphs indicate symphonies and string quartets, respectively. 


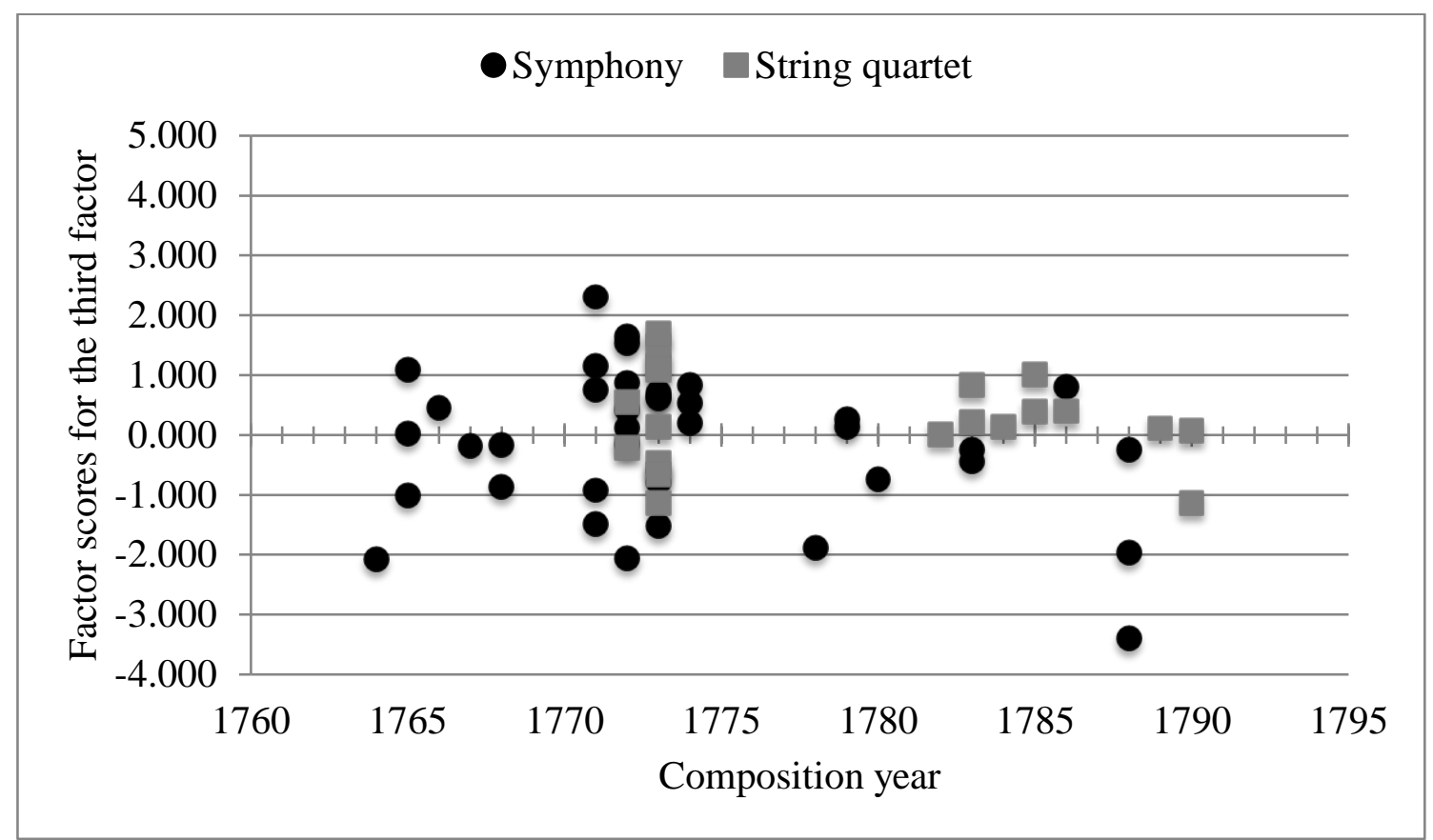

Figure 4. The relationship between composition year (horizontal axis) and the factor scores for the third factor (vertical axis). The black circles and gray squares plotted on the graphs indicate symphonies and string quartets, respectively.

\section{Discussion}

From the results of the factor analysis, the first factor appears to have a stronger relation to $I_{p n}$ (viola), $I_{d}$ (viola), $I_{p n}$ (violoncello), and $I_{d}$ (violoncello). These variables are the independencies for pitch-name and duration for either the viola or the violoncello, which are the lower two string parts. We label this first factor as the Lower part PitchName and Duration factor (LPND factor). Similarly, the second factor has a stronger relation to $I_{p c}$ (violino $I$ ), $I_{p c}$ (violoncello), $I_{p c}$ (viola), and $I_{p c}$ (violino II). These variables are the independencies of pitch-change for all parts. The second factor's label can be the Pitch-Change factor (PC factor). The third factor has a stronger relation to $I_{p n}$ (violino $\left.I\right), I_{p n}$ (violino $\left.I I\right), I_{d}$ (violino $I$ ), and $I_{d}$ (violino $I I$ ). These variables are the independencies for pitch-name and duration for violino I or violino II, which are higher parts among the four string parts. We label the third factor as the Higher part PitchName and Duration factor (HPND factor).

Figure 2 indicates that there was a fallow period for string quartets and symphonies between 1774 and 1778, and after that, a borderline between symphonies and string quartets seems to emerge. This suggests that string quartets were more affected by the LPND factor than the symphonies after the fallow period. That is, string quartets have a tendency to be composed with large values for both $I_{p n}(p)$ and $I_{d}(p)$ for the lower 
Discriminating between Mozart's Symphonies and String Quartets Based on the Degree of Independency between the String Parts

parts. In other words, the viola and the violoncello have more important roles in harmony and rhythm in string quartets. This could be a criterion that distinguishes string quartets from symphonies.

Two symphonies composed in 1788, which include positive factor scores, are K. 543 and K. 550; together with K. 551, they are known as the "last three symphonies." Although the motivations behind the composition of these three works are unknown, one hypothesis is that Mozart meant to publish them as a set, and so he may have specifically considered the degree of diversity among them (Osaki 2005). The factor scores for these three compositions would seem to be consistent with their diversity. It is possible that Mozart intentionally composed K. 543 and K. 550 to be similar to string quartets.

Figures 3 and 4, on the other hand, do not indicate any clear distinctions between the two genres. That is, the PC factor and the HPND factor do not contribute to discriminating between the two genres as much as the LPND factor does.

Moreover, works composed before 1774 could not be distinguished within this study. It is possible that there were distinctions in Mozart's mind or that another feature could reveal their differences. Given that Mozart did not compose any symphonies or string quartets between 1775 and 1777, it seems likely that he changed his way of composing during that period.

\section{Conclusion}

The purpose of this paper has been to demonstrate that the independencies between the string parts can be employed to discriminate between Mozart's symphonies and his string quartets. The results indicate a distinction between works composed after 1782 in terms of the LPND factor, which relates to the independencies of duration or pitch-name for the lower parts (viola or violoncello). It is possible to hypothesize that Mozart deliberately assigned more important roles to the lower parts in his string quartets than in the symphonies he composed during that period. 
Discriminating between Mozart's Symphonies and String Quartets Based on the Degree of Independency between the String Parts

\section{References}

Becker, Paul. 2013. Ōkesutora no ongakushi:. Translated by Tetsuya Matsumura. Tokyo: Hakusuisha.

Cambouropoulos, Emilios, Maxime Crochemore, Costas S Iliopoulos, Manal Mohamed, and Marie-France Sagot. 2005. "A Pattern Extraction Algorithm for Abstract Melodic Representations That Allow Partial Overlapping of Intervallic Categories." In Proceedings of the 6th International Conference on Music Information Retrieval (ISMIR 2005), 167-74. London. Accessed July 21, 2017. http://ismir2005.ismir.net/proceedings/1022.pdf.

Chai, Wei, and Barry Vercoe. 2001. "Folk Music Classification Using Hidden Markov Models." In Proceedings of International Conference on Artificial Intelligence, 6 (6.4).

Conklin, Darrell, and Mathieu Bergeron. 2007. "Discovery of Generalized Interval Patterns." In Proceedings of the Fourth Sound and Music Computing Conference, 149-52. Lefkada. Accessed July 21, 2017.

http://smc07.uoa.gr/SMC07\%20Proceedings/SMC07\%20Paper\%2023.pdf.

Hickman, Roger. 1981. “The Nascent Viennese String Quartet.” The Musical Quarterly 67 (2): 193-212.

Hodeir, André. 1973. Ongaku no keishiki. Translated by Hidekazu Yoshida. Tokyo: Hakusuisha.

Osaki, Shigemi. 2005. Bunka toshiteno shinfon̄ I. Tokyo: Heibonsha.

Zaslaw, Neal. 2003. Mōtsaruto no shinfōi: kontekisuto, ensou jissen, juyō. Translated by Tadashi Isoyama and Miho Nagata.Tokyo: Tokyo Shoseki. 